\title{
Getting into the mind of a worm-a personal view
}

\author{
John G. White ${ }^{\S}$ \\ Laboratory of Optical and Computational Instrumentation, University of Wisconsin, Madison WI \\ 53706, USA
}

\section{Table of Contents}

1. References 10

2. Author biography

Ever since I can remember, I liked to take things to bits to see how they work. My parents thought that this trait was not to be encouraged when applied to living animals, so diverted my attention to things mechanical and electronic. I was given the use of a shed in the garden. This became my beloved workshop where I made bombs, rockets, and radios. After one of my bombs caused a local scare, my activities were restricted to electronics. On finishing school, I worked in a factory for a time and then progressed onto a couple of other jobs in industrial development labs on the basis of my self-taught skills in electronics. Eventually, I got a job as an electronics technician in the Medical Research Council's labs in Mill Hill (MRC National Institute for Medical Research, Mill Hill, London, UK). It was here that I got my first (ethical) exposure to biology. I developed a voltage clamp apparatus for neurophysiological research, a project that piqued my curiosity about nervous systems. I also developed a computer system for displaying 3D images of molecular structures. The MRC encouraged and supported me to study for a physics degree at Brunel University. However, after I graduated in 1969, there seemed no good career path where I could pursue my developing interest in computers in the lab where I was working, so I sought and obtained a job offer from industry. However, my boss at the MRC Mill Hill lab encouraged me to check out the MRC Laboratory of Molecular Biology (LMB) in Cambridge where he had heard there was a new research initiative to use computers to study the nervous system of a worm. So, mainly out of a sense of duty to the MRC for having supported me though my undergraduate studies, I donned my best (and only) suit and headed off to Cambridge.

As soon as I walked through the door of the LMB, it was clear that this was a very different place to anything I had previously experienced. There was a buzz of feverish activity taking place, mainly from the many Americans that seemed to be around. The individual labs were crowded and the corridors were cluttered with incubators and centrifuges. An extraordinary man with bushy eyebrows wearing a striped Breton shirt interviewed me. Through clouds of cigarette smoke Sydney Brenner laid out his plans to use nematodes to study the development and function

\footnotetext{
*Edited by Paul W. Sternberg. Last revised: February 7, 2013, Published June 25, 2013. This chapter should be cited as: White J.G. Getting into the mind of a worm-a personal view (June 25, 2013), WormBook, ed. The C. elegans Research Community, WormBook, doi/10.1895/wormbook.1.158.1, http://www.wormbook.org.
}

Copyright: (C) 2013 John G. White. This is an open-access article distributed under the terms of the Creative Commons Attribution License, which permits unrestricted use, distribution, and reproduction in any medium, provided the original author and source are credited.

§ To whom correspondence should be addressed. Email: jwhite1@wisc.edu 
of nervous systems. Nematodes, I was told, have simple nervous systems that were well conserved among species. The plan was a two-pronged assault. The main effort was directed at using electron microscopy to obtain a detailed knowledge of the synaptic connections of $C$. elegans. In addition, Tony Stretton, who was introduced to me, was studying the nervous system of Ascaris lumbricoides, a nematode some 100 times larger than C. elegans that offered the potential of electrophysiological studies on neurones whose counterparts were being characterised in $C$. elegans. It soon became clear that Sydney's interest in me was because of my computer graphics programming experience, as he had ambitious plans to reconstruct the worm's nervous system from electron micrographs of serial sections using computers to input, archive and visualise the anatomical data.

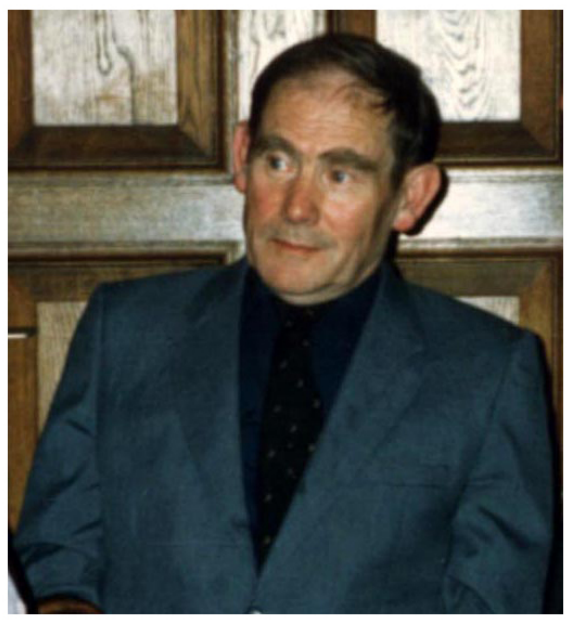

Sydney Brenner

Sydney is the most intellectually seductive person I have ever encountered. I was entranced by the possibility of obtaining a circuit diagram of a nervous system and using this to figure out how it works and assembles itself. Even so, the project seemed ridiculously ambitious and Sydney seemed a bit like the Pied Piper of Hamelin on speed-leading all who followed into the unknown. When I returned from the interview, I was still thinking of accepting an offer to work on computer-controlled telephone exchanges but could not put the thought of worms out of my mind. The more I thought about it the more intriguing the project seemed. Matters were decided by a brief telephone call from Sydney. He simply told me that it would be easy to relocate me from one MRC laboratory to another and that I should start as soon as possible. I realised that an inexorable tide of fate was carrying me along, so I just went with the flow. Thus it was that a few weeks later that I started work at the LMB without ever applying for a job there.

The LMB was a very different working environment to anything I had previously experienced. People seemed to be working around the clock and there was an all-pervading sense of excitement, which is often characteristic of new ventures. It soon became apparent that this atmosphere was particularly prevalent in the Cell Biology Division, which was jointly headed by Sydney and Francis Crick at the time. Sydney gave quite a few lectures around the world on his plans to establish $C$. elegans as a new model system. I attended one at Cambridge University. Typically, it was delivered off the cuff and was very entertaining. I remember that, having described the organism, he then spoke of an insurmountable barrier to the study of metazoans-biochemistry. He illustrated this with a bell-shaped curve on the blackboard. Then he turned to the audience with his bushy eyebrows twitching with mischief and, with a theatrical flourish, drew a line through the bottom of the curve saying: "with genetics, we will tunnel through this barrier". In this manner the Pied Piper attracted a series of outstanding American postdoctoral fellows to work on the $C$. elegans project. The MRC did not offer post-doctoral stipends, so the postdocs that came had to bring their own funding. This was challenging, as the main U.S. Federal granting agencies did not support overseas postdocs. Also, a postdoc at that time had to be particularly brave (or foolish) to work with a new model system such as the worm. There were considerable doubts as to how anything learnt from the worm could be applied to vertebrates, thereby raising the spectre that work on $C$. elegans could be unfundable when the postdocs returned to the USA to set up their labs. All these hurdles meant that the postdocs that did come over were very smart, motivated, and had a spirit of adventure. There seemed to be a synergy between the Americans and the Brits at the LMB in the early days. The Americans were bursting with energy and professionalism wanting to focus all their energies on "their" projects. The Brits seemed like gifted amateurs, having a more languid approach, enjoying extended coffee, lunch, and tea breaks discussing science. Some of the American get-up-and-go seemed to rub off 
on the Brits and the Americans learned to think a bit more about their projects. For the first few years I was at the LMB, Sydney used to hold forth at the "10 o'clock show" in our small coffee room. It was here that I started to learn some biology. During these sessions we were often entertained by Sydney's descriptions of some of the prominent figures of the time in molecular biology. "Halitosis of the Mind" was an affliction Sydney attributed to some of whom he had a particularly low opinion. I still chuckle at the notion of certain scientists breathing bad thoughts.

The MRC had built an extension to the LMB, which was completed just before I arrived. Sydney, along with several other notable molecular biologists of his generation, had been seeking a new model organism with which to tackle fundamental questions of developmental and neurobiology. He had chosen C. elegans for reasons that were reiterated in most of the early $C$. elegans publications. Crucially, for Sydney, he wanted a genetically tractable organism with a simple body plan that would facilitate detailed anatomical reconstructions from electron micrographs. To this end, he was fortunate in obtaining the services of an outstanding electron microscopist, Nichol Thomson. Nichol, a dour Scotsman, was a perfectionist who was not satisfied until he obtained stunning images. He became skilled at cutting long unbroken series of sections, a critical skill that allowed us to reconstruct the nervous system. He had been working with Sydney since the mid 1960s and they had found that C. elegans gave the nicest images of all the nematode species they examined. Sydney had started reconstructing the sensory apparatus of the head and then moved on to partially reconstructing the retro-vesicular ganglion (RVG). His technique was to use hand tracings of transparent overlays. Sydney was isolating and mapping mutants at the same time as he was undertaking these early anatomical studies. I was struck in my early days at the LMB by the incredible effort that went into establishing the genetic map of C. elegans, which saw its fruition in his 1974 Genetics paper (Brenner, 1974). In addition, Sydney partially reconstructed the RVG region in some uncoordinated mutants and identified a couple that failed to send out commissures to the dorsal cord (unc-5 and unc-6) and one where a particular nerve process was located in an aberrant position (unc-30). However, not long after I arrived, he dropped his anatomical reconstructions and moved on to pastures anew.

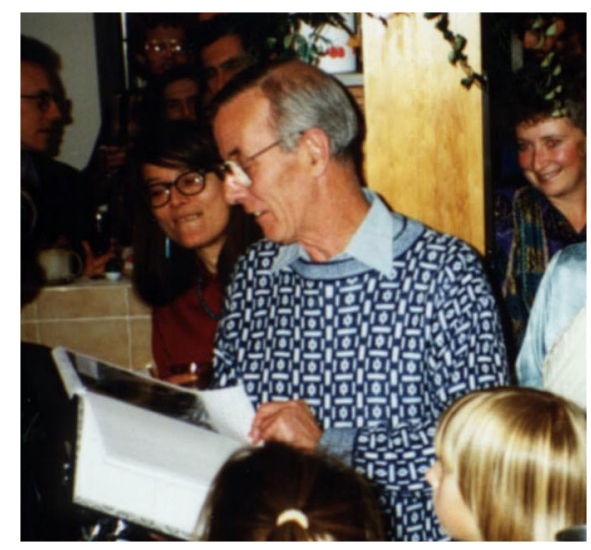

Nichol Thomson

There were three other Brits working on $C$. elegans projects when I started at the LMB. A graduate student who danced to the music of Mao Zedong and his Gang of Four was trying to trace cell lineages in the embryo, a flamboyant fellow who had recently returned from a flower-powered postdoc in California was studying behaviour, and a retiring Earth Father who had just completed a postdoc in pre-biotic chemistry was developing neurotransmitter assays. Only one stayed with the project. Initially, I was given a lab bench next to Muriel Wigby, a technician who helped Sydney with his studies of $C$. elegans genetics. Muriel taught me worm craft and I joined an on-going mutant hunt for a time. However, I soon started work on the computerized nervous system project, which rapidly became all-consuming. The plan was to digitise electron micrographs of serial sections by tracing aligned sequences of sections on a digitisation tablet. The data would then be used to generate interactive displays of the 3D structure and synaptic connections of a selected group of neurones.

In retrospect, the project was ridiculously ambitious, given the computer hardware available at the time (1970). Yet, with the courage of innocence we forged ahead, ordered a Modular 1 computer, and started work on the design and construction of the data acquisition system. Sydney conceived an ingenious design to successively align electron micrographs and transcribe them onto $35 \mathrm{~mm}$ film. I developed a system where the $35 \mathrm{~mm}$ images from a computer-controlled projector could be back-projected onto a coordinate digitiser, which could transfer to the 
computer the outlines of nerve processes drawn by hand. Fortunately the LMB had a wonderful workshop in which all these gadgets were constructed. Much of the hardware, including the coordinate digitiser, had to be made from scratch as nothing suitable was commercially available at that time.

We realised that the project we were proposing would stretch the capabilities of the computers that we could think of purchasing. We chose the Modular I, a first generation minicomputer, as it seemed the best available, particularly as it could be supplied with large (vector) graphics screens and removable storage disks. However computers at that time were pretty feeble compared with what is commonplace today. By way of comparison, the desktop computer that I am currently using has 30,000x more RAM, 70,000x more hard-disk, and runs 1000x faster than the Modular I. Perhaps even more significantly, modern computers have sophisticated operating systems and are reliable. The Modular I had no disk operating system, just simple handlers for interrupts and memory allocation. The system was controlled by a teletypewriter. Punched paper tape was used to input programs and there was no means of producing hard-copy graphical images. Most of the software we wrote used assembly language. This was laborious to write and debug but ran faster than programs written in FORTRAN II, which was the only high-level language available.

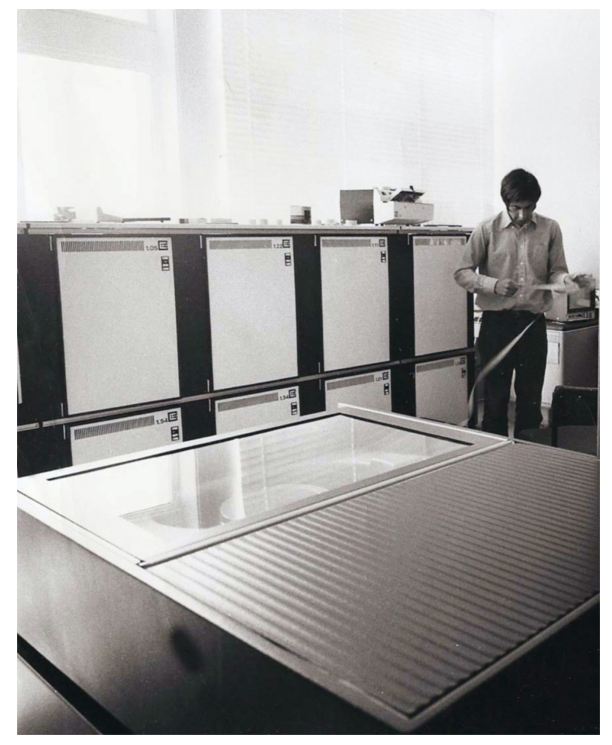

John White and the Modular I

As soon the Modular I was installed, Sydney became totally immersed with programming. Indeed, for a couple of years he seemed to do little else, often irritating people within our group who found that he was unwilling to discuss any other topic. He quickly learned assembly language and became a proficient programmer. A group in Oxford (UK) had ported the string-processing macro language TRAC onto the Modular I. Sydney set this up on our machine and soon mastered its strange syntax. For my part I realised that some basic tools were going to have to be developed for the reconstruction project to succeed. I started by writing a text editor, moved on to implementing a disk filing system, and then designed software drivers for our graphic displays. This gave us some basic tools for developing software. We realised that the TRAC language could be used to write command and control macros for an operating system, i.e., be a scripting language in modern parlance. So, after a lot of hard work, we finally developed our own graphics-based operating system. However, we were still a long way from developing anatomical reconstruction software.

After our computer system was operational, I realised that we needed three more tools: a database system for storing anatomical data, a data-efficient method of capturing coordinate data from traced neurone profiles, and a method for producing hard copies of the reconstructions. I used a rather unusual hierarchical ring-based data structure rather than the more conventional tree structures, which eventually worked quite well. The inputting system linearised the output from the digitiser and used simple data compression strategies to minimise the bulk of the coordinate data. We bought a Varian graphic plotter for which I designed an interface and wrote a software driver. Thus armed, I could finally start writing some application software. This was written in FORTRAN II. Programs were quite compact, mainly consisting of subroutine calls to operating system routines. Surprisingly, the 
application software was by far the easiest part of the whole project to implement. The final system could input sequences of aligned EM images and allow the operator to associate labels with each process. Synaptic data could also be added. The selected regions of the anatomical data that had been entered could be displayed as a rotatable 3D image or a formalised connectivity plot.

The system proved useful for visualising the 3D structure of sensory endings such as those in the head. We used it also for a few reconstructions of regions of ventral cord. Here we found the system rather less useful, as a lot of data preparation and entry was required to tell us that most of the processes in the ventral cord run as parallel bundles, something that was readily apparent from looking at sequences of printed images. By the time the computer reconstruction system was fully operational, we were focussed on reconstructing the neuropil of the nerve ring. We soon found that the only way we could make progress with this structure was to print EM images and make 4- or 6-print montages of each section of the ring. Limitations of data storage and speed meant that our computer system could never cope with data of this quantity and complexity. However, the Modular I did play a critical part in the nervous reconstruction project—checking synapse allocations. Reconstructions were accomplished by following one or a few processes at a time through successive sections, and recording when a synaptic connection was identified and at what section this occurred. Thus every synapse was recorded twice (i.e., once for the pre-synaptic process and once for the post synaptic process). We used the computer to search through these data and flag any inconsistencies, such as a missing pre- or post-synaptic partner in a specific section range. Going back to the pictures and re-scoring the synapse in question resolved these inconsistencies. Sydney pressed on with his TRAC programming and used the system to develop DNA sequence analysis procedures. Accessible computer systems with interactive computer graphics were not very common in the $70 \mathrm{~s}$, so our system got used for several unrelated studies, such as 3D reconstructions of the distribution of mitochondria in yeast and for computer modelling of phylotaxis in plants. Some years later, I found the system invaluable for modelling the dynamics of cytokinesis in animal cells when I became interested in cell division.

Although I was engrossed with programming during my first year at the LMB, I slowly learned to recognise many of the features within electron micrographs of nervous tissue. I began to think how to go about the reconstructions. Then in 1971 there was, in retrospect for me, a momentous series of events. Tony Stretton relocated to Wisconsin taking the Ascaris project with him and I inherited one of his technicians, Eileen Southgate. Eileen had been working at the LMB from its earliest days in the Cavendish Laboratory. She had recently been working with Tony reconstructing regions of the Ascaris nervous systems from light micrograph sections. She had many attributes that I lacked: she worked steadily and meticulously, she had a good visual memory, and she took careful notes of all that she did. I subsequently found out that her hobby was assembling 1000-piece jigsaw puzzles. Although our temperaments were very different, we developed a wonderfully productive working relationship, which lasted for the rest of my sojourn at the LMB. About the same time that Eileen arrived on my scene, Sydney started to wind down his own anatomical studies. This meant that I could start working with Nichol to get an extended series cut that we could use for reconstruction. Finally, in 1971 I enrolled for a PhD—seldom has a graduate student had so many amazing resources laid at their feet.

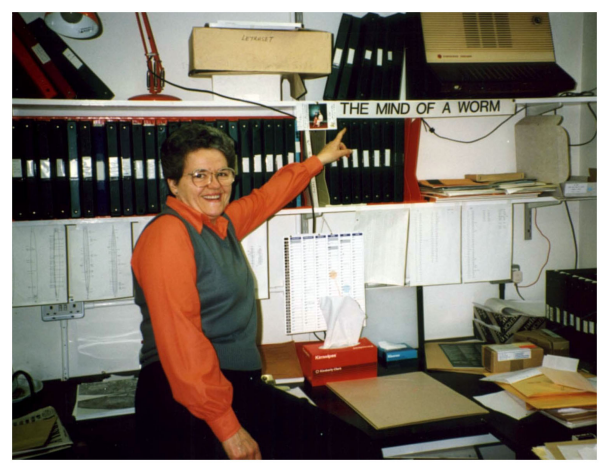

Eileen Southgate

Nichol was a remarkable electron microscopist. He was a true perfectionist, who would keep trying for weeks or months to get a specimen that gave the best quality images. He developed methodologies for sectioning very long sequences with no large gaps. Inevitably some sections get lost on grid bars when using conventional EM grids, and it soon became apparent that this would be a real problem for reconstructing the nerve ring; Nichol managed to cut two unbroken series (N2U and JSH) through the nerve ring using slot grids, which allowed virtually all sections to 
be photographed. We could not have reconstructed the neuropil in the nerve ring without these two animals. Furthermore he managed to keep sectioning the N2U series right on past the vulva, providing an invaluable data set that allowed us to reconstruct several repeats of the ventral and dorsal cord logic and to connect the ventral cord with the nerve ring. Nichol had a couple of small rooms off the main worm lab. When he was frustrated because things weren't going right, he would come out and we would commiserate with him. He acted as a sort of father figure to some of the postdocs and we grew fond of him.

Eileen and I decided to start reconstructions in the RVG using some of the series that Sydney had commissioned for his studies on this ganglion. We did not find the wax pencil on transparent overlay approach that he used to be very workable for extensive reconstructions so we sought another approach. Eventually we came up with the scheme of using Rotring coloured pens to directly label profiles on the micrographs. We found that we could erase the labels using a cotton bud soaked in alcohol. As we carried on with this work Eileen and I developed a sort of modus operandi. Eileen would photograph a series of sections in the EM and then print them. She would then follow and label individual processes or small groups. Then she would score chemical synapses and gap junctions. I would then go through these assignments with her and try to sort out ambiguities and try to make sense of the emerging reconstructions. It was soon clear that there was often more than one post-synaptic partner at a chemical synapse. Indeed several processes seemed to send out dendrites that intercepted a synapse between two other processes. Subsequently we found that this behaviour was characteristic of certain neurones, such as the D-type neurones of the ventral cord and the RMD neurones of the nerve ring, which send out a dendrite spine that dips into synapses between motorneurones and muscles. We also convinced ourselves that some other synapses that did not involve these "dippers" were also dyadic or triadic. This was based on repeated observations of the same process groupings around a synapse. Nevertheless, we did not find any unique structures on processes that were adjacent to pre-synaptic specialisations, so assigning post-synaptic processes was somewhat akin to determining guilt by association. Gap junctions were more difficult to identify than chemical synapses-we spent a lot of time scrutinising potential gap junctions. We realised that their visibility varied between animals, presumably because of slight differences in fixation and staining, and learned to adjust our criteria for identifying these organelles accordingly. Eventually we became more confident about scoring these connections, particularly when we started assembling some synaptic circuitry and saw clearly identifiable patterns of gap junction connections emerge.

Nichol and Sydney had produced a long series of sections running from the tip of the snout and covering the nerve ring (N2T). Sydney used this series to explore the sensory sensilla in the head. This project was picked up by Sam Ward, the first American postdoc to come to the LMB to work specifically on the worm project. Sam managed to link the processes of all the sensilla to their respective cell bodies and made 3D reconstructions of some of the sensory endings. This work formed the basis of the first neuroanatomical paper to come out of our group (Ward et al., 1975). A few years earlier, Dick Russell, who had been a postdoc at the LMB (although not working on $C$. elegans), set up the first offshore C. elegans lab at the California Institute of Technology. Not long after Sam's paper came out, Randle Ware, from Dick's lab, published an outline description of the head neuroanatomy (Ware et al., 1975), so we were not alone. The N2T series was a superb series and was used by Donna Albertson to obtain a complete anatomical reconstruction of a pharynx. This organ is a virtually self-contained model organism within a model organism. Donna's reconstruction yielded some interesting surprises, such as revealing a circular neurone.

Eileen and I initially focussed on reconstructing the ventral cord. As we explored further down the cord, some regularities started to emerge. We were able to define several distinct classes of motorneurone, and gave them names. The most challenging aspect of these studies was reconstructing the motorneurones that innervate the dorsal muscles. These neurones have their cell bodies in the ventral cord and send circumferential commissures round to the dorsal cord along which they run. Following the commissures all around the body was often difficult. However, when it was all sorted out some beautiful regularities emerged. We found that there are three main classes of motorneurone innervating the body musculature, A, B and D. Each class has ventral and dorsal members (VA, DA, $\mathrm{VB}, \mathrm{DB}, \mathrm{VD}$ and DD). The As and the Bs receive synaptic inputs from class specific sets of interneurone, whilst the Ds receive their synaptic inputs from the As and Bs at dyadic synapses. The VDs innervate ventral muscles and are post-synaptic to the A and B neurones on the dorsal side, whereas DDs innervate dorsal muscles receiving their synaptic input from As and Bs on the ventral side. I was excited to see this pattern emerge as it suggested that the Ds might act as cross-inhibitors. Also it seemed likely that the A and B motorneurones and their associated interneurones could be used for forward and backward locomotion.

John Sulston joined Sydney's group a few months before me. He started by developing assays for neurotransmitters with a view to identifying mutants that are deficient in particular neurotransmitters. In the course of this work, he observed that the newly hatched L1 larvae had fewer neurone cell bodies in their nerve cords than 
did later larval stages. This was an interesting observation as the dogma at the time was that there is no post-embryonic production of neurones during nematode development. John investigated the source of the extra neurones and determined the sequence of cell divisions by which they are produced. The rest of this story is, of course, history. When John first started to produce the cell lineages of the ventral cord precursors, we placed bets as to what would be the relationship between neurone type and its lineage. I won and we celebrated with a bottle of red wine. Unfortunately we did not have a corkscrew so I improvised by putting a hypodermic needle through the cork and squirting Freon through it into the wine bottle. When the cork finally came out a fountain of red wine shot up and hit the ceiling from all the liquid Freon that was boiling. The red stain stayed there for many years.

I was working on the ventral cord with Eileen one day when Sydney came in with a visitor in tow to whom I explained how we were beginning to reconstruct the ventral cord. As they were leaving, I couldn't help but hear Sydney telling the visitor that we were going to reconstruct the whole nervous system. I was rather taken aback by this remark, as, up to that moment, I had not really thought that such an undertaking was feasible. However, some time later, after we started to make some sense of the ventral cord, I gained enough confidence to begin to think seriously about reconstructing the nerve ring. We started with the N2T series, which Eileen photographed in the region of the nerve ring as four-print montages. Because the orientation of the nerve ring was close to that of the plane of sectioning, many processes were cut tangentially, which made discerning the boundaries of the membranes on adjacent processes very difficult. However, we found that this could often be achieved by reference to the images in the next and previous sections. Also, we found that the ring is a very symmetrical structure, so we could often figure out what was going on in a difficult region by looking at the symmetrically equivalent region on the other side of the nerve ring. Nevertheless, in several critical regions, the gaps in the N2T series presented insuperable barriers that we could not cross. But comparisons of the nerve rings in N2T, N2U and JSH eventually made the task possible, especially since the latter two animals were on slots grids, so we lost no sections to grid bar obscuration in this critical region. After Eileen had photographed and six-print montaged the nerve ring sections from N2U, reconstruction started in earnest on this animal. The N2U animal was an old adult that gave very clear images of neuropil.

We were excited when we found that we could trace many processes through the ring, although there were some very difficult regions. I devised a simple projection scheme that allowed us to plot the trajectory of neurones whilst retaining some spatial information. Plotting profiles proved problematic however. When sections are cut in a microtome, they suffer varying degrees of compression. This leads to varying degrees of elliptical distortion in the images. We overcame this problem by drawing a series of polar coordinate scales on a transparent overlay with different degrees of elliptical distortion. For each section, a scale was chosen that had the closest match to the ellipticity of the section. Coordinates of features of interest as measured on this scale would then be distortion free when plotted using circular polar coordinates. Nichol produced another high-quality nerve ring series sectioned on slots - the JSH animal. This was a smaller, younger animal (probably an L4), which was reconstructed along with the N2U animal. Also, we found that we could now interpret more in the N2T animal from what we were learning from the other two series and use it to resolve ambiguities. Comparing reconstructions from the different series gave us interesting insights as to the consistency of connection and branching patterns between animals.

After the nerve-rich head region, the body presented a different set of challenges. This is by far the largest region, but, apart from the ventral cord, contains little in the way of neuropil. The N2U series was key to our reconstructing the ventral and dorsal cords as it allowed us to determine the organisation of the repeating members of the motorneurone classes along the cord and to trace many of the interneurones back to the nerve ring. This series also allowed us to describe the innervation of the vulval muscles. A good quality series was cut of the hermaphrodite tail, JSE, and this allowed us to reconstruct this region from the tip of the tail to the pre-anal ganglion. The male tail is considerably more complex than that of the hermaphrodite, with its elaborate copulatory apparatus containing many additional neurones over those in the hermaphrodite. While John Sulston was determining the lineages of the male tail, Donna Albertson managed to partially reconstruct the very complex male-specific ganglia in this region using an excellent series (N2Y) that covered the posterior to the tail. We used reconstructions from the N2Y posterior ventral cord to validate that the sequence of motorneurones that had been defined in the anterior body was also present in the posterior body of the male, together with some additional neurones that are presumably used for sex-specific behaviours. So finally we had recorded nearly all of the neuropil in C. elegans on electron micrographs and had reconstructed the synaptic connections that we observed.

I have often wondered how dry stone walls are built. Once, over several days, I observed this process and came to realise that the key factor was time. Fortunately the MRC allowed us time; piece-by-piece, our stone wall was assembled over the course of some 13 years. Although progress was slow, it was generally discernable. Our motivation was maintained by the excitement of slowly uncovering new structural features and synaptic circuitry. I 
worried for a long time over how we were going to present all the data from the reconstructions in a paper. Lois Edgar, with her artist's eye and knowledge of the tail anatomy, produced some attractive 3D renderings of various parts of the nervous system. However, when we had finally obtained connectivity data for all of the 302 neurones that were identified in the hermaphrodite, we decided to present each neurone individually with a diagram of its shape annotated with its synaptic connections, as this seemed the most concise way of depicting both morphology and connectivity. I remember the huge feeling of relief I felt when the reconstruction manuscript was accepted for publication. We sent the text and figures to the publishers in a number of ring binders (no PDFs in those days). We had labelled these notebooks "the mind of a worm" in order to identify them on a shelf among all the other similar-looking notebooks. We were amused the printers had picked this up and used it as a running title, so we let the name stick. David Hall had been undertaking a parallel reconstruction of the tail of the hermaphrodite, so when this work was published, it was gratifying to see that our reconstructions were quite similar, indicating that the organisation and connectivity of neurones is largely conserved at least in this N2 strain of C. elegans. Some postdocs, notably Bob Horvitz and Ed Hedgecock, endeavoured to learn the techniques of serial section reconstruction from electron micrographs and applied them to good effect when they set up their own labs. Others chose to avoid pursuing projects that required reconstructions, possibly because of concerns about acquiring the resources needed to undertake such studies when they set up their own labs.

People have asked me what we learned from our reconstructions of the C. elegans nervous system. This obviously depends on individual perspectives. For myself, I was struck that neurones seemed to interact with adjacent members of their own class to define regions of synaptic activity. For example, the body musculature receives synaptic input at all regions along the length of the animal from the three main classes of motorneurone: A, $\mathrm{B}$, and D. However, the transition regions where one member of a class stops and the next member starts making synapses are not in register, implying that intraclass competition is defining synaptic fields. Gap junctions may be involved in this implied intraclass communication as there are many cases of classes with members that make gap junctions with each other, although chemical synapses are a rarity between members of a class. There are, of course, many other interesting features. One that I found surprising became apparent when we started looking at the neighbourhoods in which processes reside. We found that most neurones make some sort of synaptic connections to many of their neighbours, i.e., neurones tend to be highly locally connected. This organisation will inevitably lead to there being a preponderance of triangular circuit motifs, which are indeed a feature of C. elegans and other nervous systems. However, perhaps the greatest value of the reconstruction effort was in defining, at least at a structural level, what a simple nervous system is. This has hopefully provided a backdrop and a starting point for the current studies of the function and development of the C. elegans nervous system.

Back when John Sulston started to show that there were stereotyped cell lineages during development, we wondered about the relative roles of cell intrinsic and extrinsic factors in determining cell lineages. Preliminary experiments undertaken by Donna Albertson and me had indicated that it was possible to kill individual cells in $C$ elegans using a focused beam from the mercury arc lamp of a fluorescence microscope. By this time my work on the computer reconstruction system had concluded, so I could re-direct my gadget-building urges to developing a laser microbeam system purpose-built for cell ablation. The original device used a large, flash-tube pumped dye laser, which destroyed a couple of microscope objectives before it was tamed. One of the first experiments I did with the system was to ablate the two somatic cells of the four-cell gonad primordium in the L1. I was amazed to see that no vulva was formed particularly as we knew from John's lineages that the vulva does not arise from the gonad primordium. Judith Kimble picked up the project and ran with it, eventually revealing the cells involved in this inductive interaction. John also used the system to gain some useful information about the cell autonomy of certain sub-lineages. Marty Chalfie was the first to use the system for functional studies on the nervous system. Marty had been studying some neurones with distinctive microtubules (ALM, PLM, AVM, and PVM), which he identified as touch receptors. Using laser ablation, he and John showed how the touch circuitry functions and also demonstrated that the A and B type ventral cord neurones are used for controlling backwards and forwards locomotion, as we had suspected. This was an exciting time for us as it demonstrated that we could obtain useful information about the function of the $C$. elegans nervous system from ablation experiments based on the connectivity, without having to resort to neurophysiology, which is difficult to undertake in this organism. 


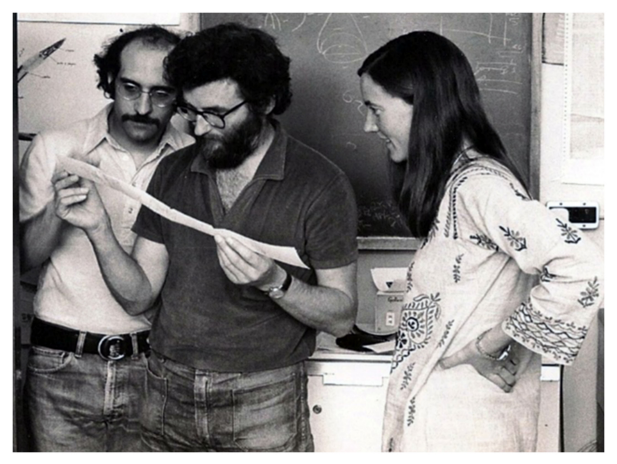

from left: Marty Chalfie, John Sulston and Donna Albertson

Early on, Jim Lewis and Jonathan Hodgkin had demonstrated that some sensilla were altered in mutants with defects in their chemotactic response. After the nervous system structure was completed, we started to explore whether uncoordinated mutants had defects in the connectivity of their nervous system, which might explain their phenotype. Serial section reconstruction of long lengths of the ventral cord is a labour-intensive way of characterising a mutant phenotype, so we selected mutants that exhibited distinctively aberrant choreographies during locomotion. We found that a few of these have specific wiring defects that can be related to their uncoordinated phenotype, although only one of these studies (unc-4) has been published. Further studies of unc-4 in David Miller's lab have gone on to reveal some of the transcription factor circuits that act to specify neurone type and synaptic specificity in the ventral cord.

John Sulston's early observation that the L1 ventral cord has only a subset of the complement of neurones present in the later stages intrigued us. As soon as we could, we reconstructed the ventral cord in an early L1 larva. It came as quite a surprise to find that the DD neurones in L1 animals innervate ventral, rather than the dorsal muscles as they do after the development of the postembryonic nervous system. Thus the DD neurones undergo a major rewiring between the L1 and L2 larval stages. Sometime later, Richard Durbin undertook an EM reconstruction study of the early development of the ventral cord in the embryo. He combined this with laser ablation experiments to explore possible interactions determining process outgrowth. These early observations and experiments went some way to demonstrate that the $C$. elegans nervous system is amenable to developmental studies.

In the late $70 \mathrm{~s}$, I started to become interested in the mechanisms for establishing polarity and the cleavage furrow in early $C$. elegans blastomeres. In 1993 I relocated to the University of Wisconsin, Madison, by which time I had become fully engrossed in these cell biology pursuits. Eileen retired soon after I left, and Nichol had retired some years previously. So, by the time I reached Madison, our involvement in nervous system reconstructions had ceased. I was concerned that the vast quantity of data that had been accumulated would be lost, as our publications could only contain a minute subset of all the images that were obtained. It seemed likely that there was a whole lot more information that could be extracted from these images than we achieved. I found a suitably large space in Madison and shipped most of the images with which I was directly involved to my new lab. Nevertheless, for a time it seemed as if this extensive collection of images would fade into obscurity. However, David Hall at the Albert Einstein College of Medicine (Bronx, NY) kept the flame of C. elegans neuroanatomy burning, and obtained a grant to set up a central repository of $C$. elegans anatomy. Scott Emmons at the same institute, had been studying male mating behaviour for some time and decided to link up with David to fully reconstruct the nervous system in the male tail. This fertile collaboration led to another grant for software development that will facilitate future reconstructions. I was delighted that these initiatives had been made and was only too pleased to let them have all our image data and notes. Much of these data together with their own and that from other labs, have been scanned and made available online, a formidable achievement. The image repository developed into the WormAtlas, which is now an invaluable resource for all investigators studying the C. elegans nervous system.

In retrospect, $C$ elegans was a shrewd choice of organism with which to study nervous system connectivity at the ultrastructural level, given its small complement of neurones each having little or no branching. Furthermore, this nematode is small enough for large regions of the body to be sectioned in an unbroken series for visualisation by electron microscopy. Recently, there has been a resurgence of interest in determining the connectivity of more complex nervous systems using improved electron microscopy and computer visualisation techniques. This will 
surely lead to exciting new insights into nervous system function and development. Indeed, the field has now gained acceptance as a respectable scientific pursuit by becoming an "-omic"-connectomics. How glad I am that I responded to the siren sound of the Pied Piper all those years ago.

\section{References}

Brenner, S. (1974). The genetics of Caenorhabditis elegans. Genetics 77, 71-94.Abstract

Ward, S., Thomson, N., White, J.G., and Brenner, S. (1975). Electron microscopical reconstruction of the anterior sensory anatomy of the nematode Caenorhabditis elegans. J. Comp. Neurol. 160, 313-37.Abstract

Ware, R.W., Crossland, K., Russell, R.L., and Clark, D. (1975). The nerve ring of the nematode C. elegans: sensory input and motor output. J. Comp. Neurol. 162, 71-110.

White, J.G., Southgate, E., Thomson, J.N., and Brenner, S. (1986). The structure of the nervous system of the nematode Caenorhabditis elegans. Philos. Trans. R. Soc. Lond. B Biol. Sci. 314, 1-340.AbstractArticle

\section{Author biography}

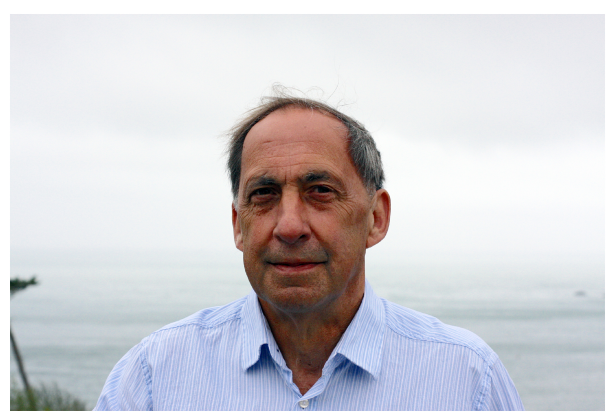

John White was born in Wales in 1943, and moved with his parents to west London after WWII. He became interested in science and technology at an early age and experimented with electronics, bombs and rockets at home. In 1962 John left school and took several engineer/technician jobs, eventually ending up in the Medical Research Council's National Institute for Medical Research in 1964. He was awarded a scholarship for a "sandwich" degree (alternating six months work, six months college) at Brunel University in 1965. After graduation in 1969 he relocated to the MRC's Molecular Biology Laboratory in Cambridge and joined Sydney Brenner's C. elegans research group. His graduate work involved setting up a computer system for neuroanatomical reconstructions, which helped him reconstruct the ventral nerve cord of C. elegans. After obtaining his PhD in 1974 he continued with the nervous system reconstruction project in Cambridge, and also became involved in laser ablation studies. Following the submission of the C. elegans anatomy paper in 1985 (White et al., 1986), he moved on to analysing ventral cords of uncoordinated mutants and then to developmental EM studies of the vulva. His growing interest in cellular dynamics, particular in regard to cytokinesis, stimulated the development of a confocal microscope to facilitate the observation of intracellular architecture. Professor White relocated to the University of Wisconsin at Madison in 1993 where his C. elegans studies focused on cytokinesis and polarity. He also headed a microscopy lab in Madison where instrumentation for observing sub-cellular dynamics using multiphoton microscopy was developed. He retired in 2008.

All WormBook content, except where otherwise noted, is licensed under a Creative Commons Attribution License. 